
\title{
Reseacch Soutere \\ Causal Effects of Life Course Adiposity on Chronic Kidney Disease: A Mendelian Randomization Study
}

\section{Wanchun Yang}

Sichuan University West China Hospital

\section{Hongchun Chen}

Neijiang Hospital of Traditional Chinese Medicine

\section{Qiuyun Yuan}

Sichuan University West China Hospital

Yang Zou ( $\nabla$ zouyang@med.uestc.edu.cn )

Sichuan Provincial People's Hospital: Sichuan Academy of Medical Sciences and Sichuan People's Hospital

\section{Research}

Keywords: Chronic kidney disease, Life course adiposity, Mendelian randomization

Posted Date: August 10th, 2021

DOI: https://doi.org/10.21203/rs.3.rs-746367/v1

License: (c) (1) This work is licensed under a Creative Commons Attribution 4.0 International License. Read Full License 


\section{Abstract}

Background Obesity is reported to be tightly correlated the development of chronic kidney disease (CKD). However, whether there exists causation is unknown, and it remains controversial about the role of obesity in CKD is protective or destructive. In this study, we try to infer the causal relationship between life course adiposity and CKD, to provide a rationale for obesity management in CKD patients.

Methods A two-sample Mendelian randomization (MR) analysis was conducted to explore the causal relationship of life course adiposity traits including including body mass index (BMI), childhood BMI, body fat percentage (BF), birth weight (BW), waist circumference, hip circumference and waist-to-hip ratio (WHR) to CKD. Significant single nucleotide polymorphisms from genome-wide association study on human adiposity traits were utilized as exposure instruments, and summary statistics of CKD as outcome. The causal relationship was evaluated by inverse variance weighted, MR Egger regression and weighted median methods, and further verified by extensive sensitivity analyses.

Results Genetically determined one standard deviation increase in adult BMI was associated with higher risk of CKD in all four MR methods. And other indexes including childhood BMI, body fat percentage, and waist/hip circumference also have a causal effect on the risk of CKD. The results were robust under all sensitivity analyses.

Conclusions There exist causal effect of life course adiposity on the risk of CKD. A genetic predisposition to higher adult BMI may increase the risk of CKD.

\section{Introduction}

Chronic kidney disease (CKD) is a global public health problem, characterized by persistent alterations in kidney structure and function(1). CKD is tightly associated with the development of end-stage renal disease (ESRD) and cardiovascular disease(2). Because CKD arises from many heterogeneous disease pathways, the underlying mechanism for CKD is complex and probably multifactorial(3). Current evidence implicates a plethora of risk factors involved in the predisposition and development of CKD, including obesity $(4,5)$.

Obesity is a widely recognized risk factor that contributes to the development of CKD(6). Compelling observational studies have provided extensive evidence for the correlative relationship between obesity and CKD, suggesting that obesity is associated with the higher risk of CKD. For example, longitudinal cohort studies and case-control studies indicated that higher body mass index (BMI) may contribute to an increased risk for CKD, indicating that early life adiposity, such as birth weight (BW) and childhood BMI, might be a long-term modifiable factor for the onset of $\operatorname{CKD}(7)$. Studies also demonstrated that increased waist-to-hip ratio (WHR) increased the risk of CKD mortality(8). Although these population-based studies reported a positive association between obesity/adiposity and CKD, conflicting evidence showed that obesity was paradoxically associated with greater survival once CKD is onset $(9,10)$. Nevertheless, all these observational studies are influenced by the possibility of confounding factors and/or reverse 
causation. Therefore, whether life course obesity/adiposity, taken as a whole, has a causal effect on the risk of CKD remains largely unknown.

To evaluate the causal relationship between life course adiposity and CKD, we employed the Mendelian randomization (MR) approach, a genetic epidemiological method to explore the causal relationship between exposures (risk factors) and outcomes (diseases) $(11,12)$. The MR approach is widely used to identify risk factors and causal associations in human diseases. Here, we chose the single nucleotide polymorphism (SNP) data from large genome-wide association study (GWAS) on hematological traits as instrumental variables for the exposure. By MR approach, we demonstrate that several indexes of life course adiposity, particularly adult BMI, were causally associated with the increased risk of CKD.

\section{Methods}

\section{Datasets}

We conducted MR analysis for seven life course adiposity traits including BMI, childhood BMI, body fat percentage (BF\%), birth weight (BW), waist circumference, hip circumference and waist-to-hip ratio (WHR) based on summarized association results from published genome-wide association studies (GWAS) with the most recent publication dates and the largest sample sizes. Genetic variants which passed generally accepted genome-wide significance threshold $(P<5.00 \mathrm{E}-8)$ were utilized as instrument variants, so that the relevance assumption of MR was satisfied. Instrument variables were clumped based on 1000 Genomes Project linkage disequilibrium (LD) structure and independent SNPs $\left(R^{2}<0.001\right.$ with any other SNP within $10 \mathrm{Mb}$ ) with the most significant P-value were retained.

Instrument variables (IVs) for BMI were drawn from the published GWAS meta-analysis involving 339,224 individuals of European ancestry(13). BMI was defined as the body mass divided by the square of body height. The units of BMI are kilograms per square meter. IVs for childhood BMI were identified from 47,541 European ancestry children(14). The childhood age ranges from 2 to 10 years old. The units of childhood BMI are kilograms per square meter. IVs for body fat were obtained from a GWAS metaanalysis involving 89,297 individuals of European ancestry(15). BF\% was defined as the total fat mass divided by the total body mass, multiplied by 100 , and was measured either with bioimpedance analysis or dual energy X-ray absorptiometry. IVs for birth weight were identified from a multi-ancestry GWAS including up to 153,781 individuals(16). BW is the body weight of a baby at birth collected from obstetric records, medical registers or interviews with the mother and self-report as adults. The unit of BW is grams. For WHR, waist circumference and hip circumference measures were obtained from a GWAS on 224,459 individuals of European ancestry(17). WHR is the dimensionless ratio of the circumference of the waist to that of the hips measured with a portable stadiometer.

Summary statistics of CKD were drawn from published GWAS meta-analysis of estimated glomerular filtration rate (eGFR) involving 1,046,070 individuals of European ancestry(18). CKD was defined as eGFR 


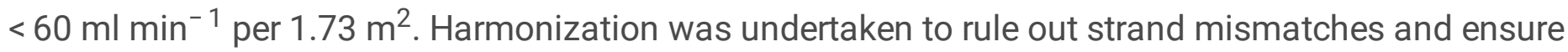
alignment of SNP effect sizes.

\section{Mendelian randomization analysis}

We hypothesized that each trait as risk factor could causally increase the risk of CKD, and the following assumptions were satisfied in the MR analysis: the genetic variants used as instrumental variables are associated with the risk factor; the genetic variants are not associated with any confounders; and genetic variants are associated with CKD through the risk factor only.

We performed a two-sample MR analysis to estimate the effect of each trait on CKD with Inverse variance weighted (IVW) method. Since IVW was not sensitive to horizontal pleiotropy of instrument SNPs, another three methods including Mendelian randomization Egger regression, Weighted mode, Weighted median were conducted as a supplement. In addition, extensive complementary sensitivity analyses were performed to evaluate potential violations of the model assumptions in MR analysis. We (i) conducted Mendelian Randomization Pleiotropy RESidual Sum and Outlier (MR-PRESSO) analysis to explore presence of outliers that could bias the results(19), (ii) evaluated the directional pleiotropy of instruments with MR-Egger regression methods, (iii) evaluated reverse causal inference with Steiger analysis(20) to check whether CKD has a causal effect on each trait, and (iv) checked heterogeneity with the Cochran Q test.

To estimate bias from sample overlap, we computed the F-statistic of each SNP to evaluate the strongness of selected instrument variables(21). We also performed a leave-one-out analysis with the Inverse variance weighted method to check whether the overall estimate was driven by single SNP. A Pvalue below $0.008(0.05 / 7)$ should be considered statistically significant after Bonferroni correction for each trait. A P-value between 0.007 and 0.05 implies a suggestive association. The main statistical analyses were conducted using $\mathrm{R}$ package TwoSampleMR(21). This study only utilized publicly available summarized results from published genome-wide association studies. No individual-level data were involved.

\section{Results}

To explore the causal relationship of life course obesity/adiposity traits on the risk of CKD, we enrolled seven life course adiposity related traits including BMI, childhood BMI, body fat percentage, birth weight, waist circumference, hip circumference and waist-to-hip ratio (WHR) for the analysis of association with CKD using four MR methods.

Results of MR analysis showed that each one standard deviation (1-SD) increase in BMI was associated with higher risk of CKD consistently in four methods, including IVW (OR: 1.214, 95\% Cl: 1.115-1.321, P: 7.9E-06), MR Egger (OR: 1.245, 95\% Cl: 1.011-1.533, P: 4.2E-02), Weighted mode (OR: 1.448, 95\% Cl: 1.202-1.745, P: 2.1E-04) and Weighted median (OR: 1.336, 95\% Cl: 1.184-1.508, P: 2.7E-06) (Fig. 1). The scatter and funnel plot displays symmetric pattern of effect size variation around the point estimated 
(Figs. 2 and 3). And 1-SD increase in childhood BMI was also associated with higher risk of CKD at nominal significant level in IVW method (OR: 1.166, 95\% Cl: 1.056-1.286, P: 2.3E-03). It's noted that the other three methods did not show significant association after the Bonferroni correction, but the effect of direction trended the same. Interestingly, the effect of childhood BMI was lower than that of adult BMI consistently in the four MR methods, suggesting the adult BMI may have larger influence on CKD risk than childhood BMI.

We also found that 1-SD increase in body fat was associated with higher CKD risk in Weighted median method (OR: 1.556, 95\% Cl: 1.193-2.029, P: 1.1E-03) and Weighted mode method (OR: 1.797, 95\% Cl: 1.307-2.469, P: 5.64E-03), and MR Egger methodshowed suggestive association (Fig. 1). But no association was found between birth weight and CKD risk (Fig. 1). Moreover, it's noted a suggestive positive association between waist circumference (OR: 1.270, 95\% Cl: 1.105-1.458, P: 7.4E-04), hip circumference (OR: 1.222, 95\% Cl: 1.076-1.387, P: 1.99E-03) and CKD risk. And similar results were found in Weighted median and Weighted mode methods (Fig. 1). However, no significant association was observed between WHR and CKD risk.

Finally, we performed extensive sensitivity analysis to validate the causal association between each trait and CKD. No heterogeneity of effects was detected using Cochran's $Q$ test (Table 1 ). The F statistics of all the instrument variables were above 10 (ranging from 19.45 to 447), indicating absence of weakness in the selected instruments. The intercept of MR-Egger is not significantly deviated from zero, suggesting no apparent horizontal pleiotropy (Table 1). Directionality examination by Steiger analysis did not suggest violation of the causality either. The MR-PRESSO analysis detected potential instrumental outliers at the nominal significance level of 0.05 , but removing the outlier did not lead to a substantial change of the causal effect. The leave-one-out results suggest that no single instrumental variable can influence the estimated causal effect. 
Table 1

Heterogeneity and horizontal pleiotropy analyses results.

\begin{tabular}{|lllllll|}
\hline \multirow{2}{*}{ Trait } & \multicolumn{5}{c}{ Heterogeneity } & \multicolumn{5}{l|}{ Horizontal pleiotropy } \\
\cline { 2 - 7 } & IVW Q & IVW Q df & IVW P value & Egger intercept & SE & P value \\
\hline BMI & 104.8 & 77 & 0.019 & $-7.70 \mathrm{E}-04$ & $2.95 \mathrm{E}-03$ & 0.79 \\
\hline BF & 30.9 & 9 & $3.06 \mathrm{E}-04$ & $-4.20 \mathrm{E}-02$ & $1.80 \mathrm{E}-02$ & 0.05 \\
\hline birth weight & 71.5 & 44 & 0.005 & $6.60 \mathrm{E}-03$ & $5.53 \mathrm{E}-03$ & 0.24 \\
\hline childhood BMI & 40.0 & 21 & 0.007 & $-3.45 \mathrm{E}-04$ & $9.41 \mathrm{E}-03$ & 0.97 \\
\hline Hip circumference & 118.6 & 50 & $1.69 \mathrm{E}-07$ & $-4.87 \mathrm{E}-03$ & $5.92 \mathrm{E}-03$ & 0.41 \\
\hline Waist circumference & 85.2 & 39 & $2.71 \mathrm{E}-05$ & $-1.64 \mathrm{E}-03$ & $5.61 \mathrm{E}-03$ & 0.77 \\
\hline WHR & 54.4 & 35 & 0.019 & $-1.71 \mathrm{E}-02$ & $8.61 \mathrm{E}-03$ & 0.06 \\
\hline $\begin{array}{l}\text { SE, standard error; IVW, Inverse variance weighted; Q, Cochran's Q test estimate; df, Cochran's Q test } \\
\text { degrees of freedom. }\end{array}$
\end{tabular}

\section{Discussion}

It's long been appreciated that obesity is a risk factor in the development of CKD through observational studies, but the causation of this association is unknown mainly due to ethical issues. Here, using a comprehensive two-sample MR analysis, we show that increased life course adiposity is causally associated with increased risk of CKD. We demonstrate that adult/childhood BMI, body fat and hip/waist circumference, but not birth weight and WHR, have a causal effect on the risk of CKD. To the best of our knowledge, this study is the first exploration that attempts to illuminate the directional causal relationship between life course obesity/adiposity and CKD, through a genetic approach based on summary statistics.

Currently, a large body of epidemiologic evidence has revealed that obesity might be a significant risk factor for CKD because of its strong link with type 2 diabetes and hypertension, the two major causes of CKD(22). Indeed, obesity-induced hypertension, hyperglycemia, hyperlipidemia, and other metabolic alternations are all potential risk factors for $\operatorname{CKD}(23)$. These findings are also supported by the fact that weight-loss strategies by either lifestyle intervention or bariatric surgery are associated with decreased risk of kidney failure in CKD patients(24). Thus, lower adiposity composition seems to be protective in CKD patients. However, controversial results showed that weight loss during the course of CKD was associated with a substantially higher risk for death, especially after dialysis therapy initiation(25). Therefore, it is still not clear whether adiposity composition exhibit a protective or destructive role in CKD development. Moreover, these observational studies could be influenced by the possibility of confounding factors. In this study, using genetic variants as proxies for each trait by Mendelian randomization (MR) approach, we demonstrate that increased life course adiposity increases the risk of CKD. 
Our findings reveal that some of the indexes of adiposity, including adult and childhood BMI, body fat percentage, and waist/hip circumference, have a causal effect on the risk of CKD. Particularly, the adult BMI has a larger influence than other factors in our MR study. This might be interpreted as that adult adiposity has a larger influence on CKD. In addition, there was weak evidence of a causal association between childhood BMI and CKD. On the other hand, we found that birth weight and waist-to-hip ratio (WHR) were not found to be causally associated with CKD in our MR study, like waist circumference and hip circumference.

There are strengths in our study, including the evaluation of life course adiposity on CKD, and the use of data from large GWASs of adiposity. Our design technique tried to minimize confounding bias with several MR methods and extensive sensitivity analysis. However, some limitations merit consideration. We chose genetic variants for the exposure from a large sample size study, but weak instrument bias cannot be fully ruled out. Moreover, population stratification and potential sample overlap might be another source of bias, as in all MR analyses.

\section{Conclusions}

In conclusion, based on our two-sample MR analysis, we demonstrate the causal association of life course adiposity, particularly adult BMI, on the increased risk of CKD. Our work expands current understandings to the relationship between obesity and CKD, and provides the rationale for obesity management to reduce the risk of CKD.

\section{Abbreviations}

CKD: chronic kidney disease,

MR: Mendelian randomization,

BMI: body mass index,

BF: body fat percentage,

BW: birth weight,

WHR: waist-to-hip ratio,

GWAS: genome-wide association study,

IVs: Instrument variables,

IVW: Inverse variance weighted.

\section{Declarations}




\section{Ethical Approval and Consent to participate}

The present study was approved by the Ethics Committee of Sichuan Academy of Medical Science and Sichuan Provincial People's Hospital (Sichuan, China).

\section{Consent for publication}

Not applicable.

\section{Availability of data and materials}

The GWAS summary statistics used to perform the analyses described in the study were obtained from publicly available published data.

\section{Competing interests}

The authors have no competing interests to declare.

\section{Funding}

This work was supported by the funding of Chinese Hospital Association Foundation (No. CHABP201612) and Wu Jieping Medical Foundation (No. 320.6750.16193).

\section{Authors' contributions}

(I) Conception and design: Wanchun Yang, Hongchun Chen and Yang Zou, (II) Administrative support: Yang Zou, (III) Provision of study materials or patients: Wanchun Yang, Hongchun Chen, Qiuyun Yuan, (IV) Collection and assembly of data: Wanchun Yang, Hongchun Chen, Qiuyun Yuan, (V) Data analysis and interpretation: Wanchun Yang, Hongchun Chen and Yang Zou, (VI) Manuscript writing: Yang Zou, (VII) Final approval of manuscript: All authors.

\section{Acknowledgments}

We thank the investigators who made the GWAS summary data publicly available and the participants who contributed to those studies. The corresponding author attests that all listed authors meet the authorship criteria and that no others meeting the criteria have been omitted.

\section{Authors' information}

${ }^{1}$ State Key Laboratory of Biotherapy, West China Hospital, Sichuan University, Chengdu 610041, People's Republic of China.

${ }^{2}$ Neijiang Hospital of Traditional Chinese Medicine, Neijiang 641000, People's Republic of China. 
${ }^{3}$ Department of Nephrology, School of Medicine, Sichuan Academy of Medical Science and Sichuan Provincial People's Hospital, Sichuan Clinical Research Center for Kidney Diseases, University of Electronic Science and Technology of China, Chengdu 610072, China.

\section{References}

1. Jha V, Garcia-Garcia G, Iseki K, Li Z, Naicker S, Plattner B, et al. Chronic kidney disease: global dimension and perspectives. Lancet. 2013 Jul 20,382(9888):260-72. PubMed PMID: 23727169.

2. Romagnani P, Remuzzi G, Glassock R, Levin A, Jager KJ, Tonelli M, et al. Chronic kidney disease. Nature reviews Disease primers. 2017 Nov 23,3:17088. PubMed PMID: 29168475.

3. Ruiz-Ortega M, Rayego-Mateos S, Lamas S, Ortiz A, Rodrigues-Diez RR. Targeting the progression of chronic kidney disease. Nature reviews Nephrology. 2020 May,16(5):269-88. PubMed PMID: 32060481.

4. Luyckx VA, Tuttle KR, Garcia-Garcia G, Gharbi MB, Heerspink HJL, Johnson DW, et al. Reducing major risk factors for chronic kidney disease. Kidney international supplements. 2017 Oct,7(2):71-87. PubMed PMID: 30675422. Pubmed Central PMCID: 6341126.

5. Garofalo C, Borrelli S, Minutolo R, Chiodini P, De Nicola L, Conte G. A systematic review and metaanalysis suggests obesity predicts onset of chronic kidney disease in the general population. Kidney international. 2017 May,91(5):1224-35. PubMed PMID: 28187985.

6. Wickman C, Kramer H. Obesity and kidney disease: potential mechanisms. Seminars in nephrology. 2013 Jan,33(1):14-22. PubMed PMID: 23374890.

7. Chang Y, Ryu S, Choi Y, Zhang Y, Cho J, Kwon MJ, et al. Metabolically Healthy Obesity and Development of Chronic Kidney Disease: A Cohort Study. Annals of internal medicine. $2016 \mathrm{Mar}$ 1,164(5):305-12. PubMed PMID: 26857595.

8. Elsayed EF, Sarnak MJ, Tighiouart H, Griffith JL, Kurth T, Salem DN, et al. Waist-to-hip ratio, body mass index, and subsequent kidney disease and death. American journal of kidney diseases : the official journal of the National Kidney Foundation. 2008 Jul,52(1):29-38. PubMed PMID: 18511168. Pubmed Central PMCID: 4052757.

9. Rhee CM, Ahmadi SF, Kalantar-Zadeh K. The dual roles of obesity in chronic kidney disease: a review of the current literature. Current opinion in nephrology and hypertension. 2016 May,25(3):208-16. PubMed PMID: 26999023. Pubmed Central PMCID: 5926196.

10. Kalantar-Zadeh K, Rhee CM, Amin AN. To legitimize the contentious obesity paradox. Mayo Clinic proceedings. 2014 Aug,89(8):1033-5. PubMed PMID: 25092364. Pubmed Central PMCID: 6040818.

11. Lawlor DA, Harbord RM, Sterne JA, Timpson N, Davey Smith G. Mendelian randomization: using genes as instruments for making causal inferences in epidemiology. Statistics in medicine. $2008 \mathrm{Apr}$ 15,27(8):1133-63. PubMed PMID: 17886233.

12. Davey Smith G, Hemani G. Mendelian randomization: genetic anchors for causal inference in epidemiological studies. Human molecular genetics. 2014 Sep 15,23(R1):R89-98. PubMed PMID: 
25064373. Pubmed Central PMCID: 4170722.

13. Locke AE, Kahali B, Berndt SI, Justice AE, Pers TH, Day FR, et al. Genetic studies of body mass index yield new insights for obesity biology. Nature. 2015 Feb 12,518(7538):197-206. PubMed PMID: 25673413. Pubmed Central PMCID: 4382211.

14. Felix JF, Bradfield JP, Monnereau C, van der Valk RJ, Stergiakouli E, Chesi A, et al. Genome-wide association analysis identifies three new susceptibility loci for childhood body mass index. Human molecular genetics. 2016 Jan 15,25(2):389-403. PubMed PMID: 26604143. Pubmed Central PMCID: 4854022.

15. Lu Y, Day FR, Gustafsson S, Buchkovich ML, Na J, Bataille V, et al. New loci for body fat percentage reveal link between adiposity and cardiometabolic disease risk. Nature communications. 2016 Feb 1,7:10495. PubMed PMID: 26833246. Pubmed Central PMCID: 4740398.

16. Horikoshi M, Beaumont RN, Day FR, Warrington NM, Kooijman MN, Fernandez-Tajes J, et al. Genomewide associations for birth weight and correlations with adult disease. Nature. 2016 Oct 13,538(7624):248-52. PubMed PMID: 27680694. Pubmed Central PMCID: 5164934.

17. Shungin D, Winkler TW, Croteau-Chonka DC, Ferreira T, Locke AE, Magi R, et al. New genetic loci link adipose and insulin biology to body fat distribution. Nature. 2015 Feb 12,518(7538):187-96. PubMed PMID: 25673412. Pubmed Central PMCID: 4338562.

18. Wuttke M, Li Y, Li M, Sieber KB, Feitosa MF, Gorski M, et al. A catalog of genetic loci associated with kidney function from analyses of a million individuals. Nature genetics. 2019 Jun,51(6):957-72. PubMed PMID: 31152163. Pubmed Central PMCID: 6698888.

19. Verbanck M, Chen CY, Neale B, Do R. Detection of widespread horizontal pleiotropy in causal relationships inferred from Mendelian randomization between complex traits and diseases. Nature genetics. 2018 May,50(5):693-8. PubMed PMID: 29686387. Pubmed Central PMCID: 6083837.

20. Hemani G, Tilling K, Davey Smith G. Orienting the causal relationship between imprecisely measured traits using GWAS summary data. PLoS genetics. 2017 Nov,13(11):e1007081. PubMed PMID: 29149188. Pubmed Central PMCID: PMC5711033. Epub 2017/11/18. eng.

21. Hemani G, Zheng J, Elsworth B, Wade KH, Haberland V, Baird D, et al. The MR-Base platform supports systematic causal inference across the human phenome. eLife. 2018 May 30,7. PubMed PMID: 29846171. Pubmed Central PMCID: 5976434.

22. Kramer H, Luke A, Bidani A, Cao G, Cooper R, McGee D. Obesity and prevalent and incident CKD: the Hypertension Detection and Follow-Up Program. American journal of kidney diseases : the official journal of the National Kidney Foundation. 2005 Oct,46(4):587-94. PubMed PMID: 16183412.

23. Foster MC, Hwang SJ, Larson MG, Lichtman JH, Parikh NI, Vasan RS, et al. Overweight, obesity, and the development of stage 3 CKD: the Framingham Heart Study. American journal of kidney diseases : the official journal of the National Kidney Foundation. 2008 Jul,52(1):39-48. PubMed PMID: 18440684. Pubmed Central PMCID: 2531220.

24. Chintam K, Chang AR. Strategies to Treat Obesity in Patients With CKD. American journal of kidney diseases : the official journal of the National Kidney Foundation. 2020 Oct 16. PubMed PMID: 
33075388.

25. Ku E, Kopple JD, Johansen KL, McCulloch CE, Go AS, Xie D, et al. Longitudinal Weight Change During CKD Progression and Its Association With Subsequent Mortality. American journal of kidney diseases : the official journal of the National Kidney Foundation. 2018 May,71(5):657-65. PubMed PMID: 29217305. Pubmed Central PMCID: 5915916.

\section{Figures}

\section{Risk factors}

BM

MR Egger

Weighted median

Inverse variance weighted

Weighted mode

Body fat

MR Egger

Weighted median

Inverse variance weighted

Weighted mode

Birth weight

MR Egger

Weighted median

Inverse variance weighted

Weighted mode

Childhood BMI

MR Egger

Weighted median

Inverse variance weighted

Weighted mode

Hip circumference

MR Egger

Weighted median

Inverse variance weighted

Weighted mode

\section{Waist circumference}

MR Egger

Weighted median

Inverse variance weighted

Weighted mode

WHR

MR Egger

Weighted median

Inverse variance weighted

Weighted mode
OR $(95 \% \mathrm{CI}) \quad$ P value

$1.245(1.011-1.533) \quad 4.24 \mathrm{e}-02$

$1.336(1.184-1.508) \quad 2.73 e-06$

$1.214(1.115-1.321) \quad 7.91 \mathrm{e}-06$

$1.448(1.202-1.745) \quad 2.12 \mathrm{e}-04$

$5.079(1.781-14.485) \quad 1.61 \mathrm{e}-02$

$1.556(1.193-2.029) \quad 1.09 \mathrm{e}-03$

$1.303(0.971-1.747) \quad 7.74 \mathrm{e}-02$

1.797 (1.307 - 2.469) 5.64e-03

$0.768(0.549-1.074) \quad 1.29 \mathrm{e}-01$

$0.894(0.781-1.023) \quad 1.04 \mathrm{e}-01$

$0.891(0.799-0.993) \quad 3.71 \mathrm{e}-02$

$0.902(0.737-1.104) \quad 3.21 \mathrm{e}-01$

$1.172(0.844-1.629) \quad 3.55 \mathrm{e}-01$

$1.158(1.030-1.303) \quad 1.41 \mathrm{e}-02$

$1.166(1.056-1.286) \quad 2.26 \mathrm{e}-03$

$1.327(0.979-1.799) \quad 8.29 \mathrm{e}-02$

$1.421(0.970-2.083) \quad 7.75 \mathrm{e}-02$

$1.214(1.043-1.413) \quad 1.23 \mathrm{e}-02$

$1.222(1.076-1.387) \quad 1.99 \mathrm{e}-03$

$1.497(1.200-1.867) \quad 7.73 \mathrm{e}-04$

$1.336(0.922-1.936) \quad 1.34 \mathrm{e}-01$

$1.406(1.197-1.651) \quad 3.36 \mathrm{e}-05$

$1.270(1.105-1.458) \quad 7.39 \mathrm{e}-04$

$1.464(1.206-1.778) \quad 4.27 e-04$

$1.641(0.903-2.981) \quad 1.13 e-01$

$0.935(0.800-1.092) \quad 3.94 \mathrm{e}-01$

$0.908(0.798-1.034) \quad 1.44 \mathrm{e}-01$

$0.991(0.752-1.305) \quad 9.48 \mathrm{e}-01$ 
Figure 1

Mendelian randomization analysis results. Forest plot showing results from the Mendelian randomization (MR) analysis to evaluate potential causal associations between obesity traits and CKD. Estimates are per 1 standard deviation increase in the trait.

A

E
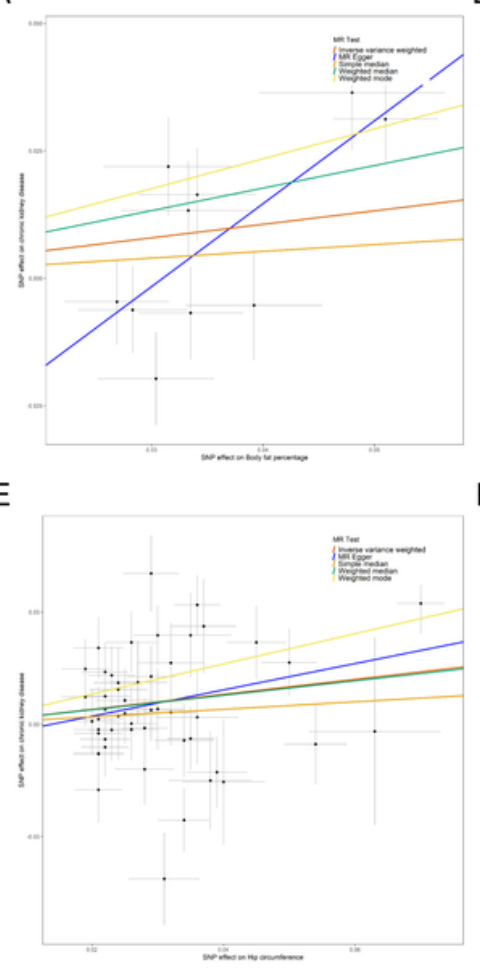

B

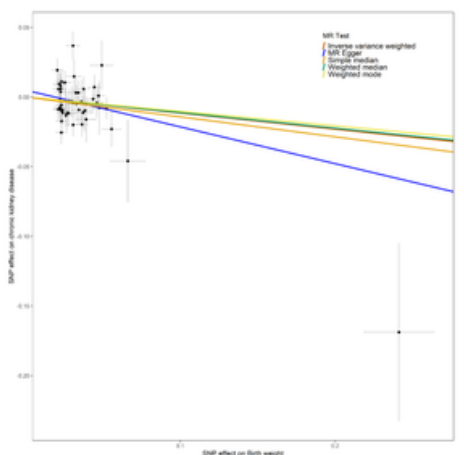

$\mathrm{F}$

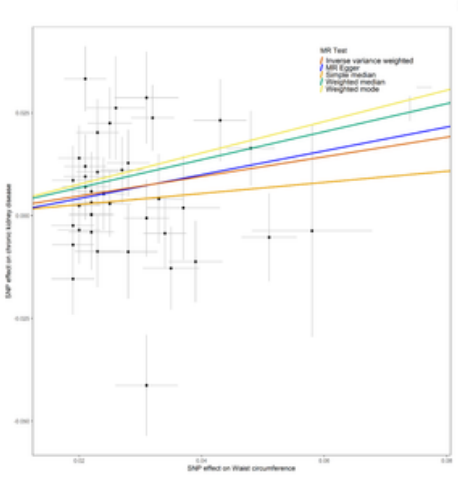

C

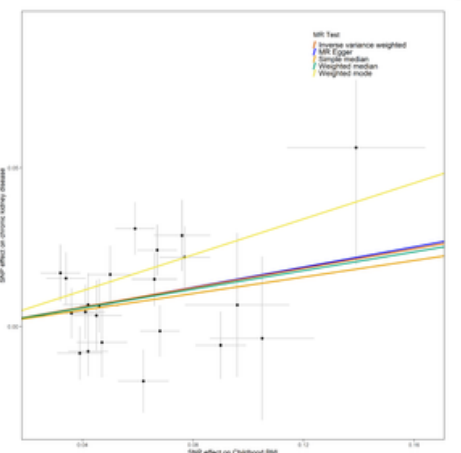

G

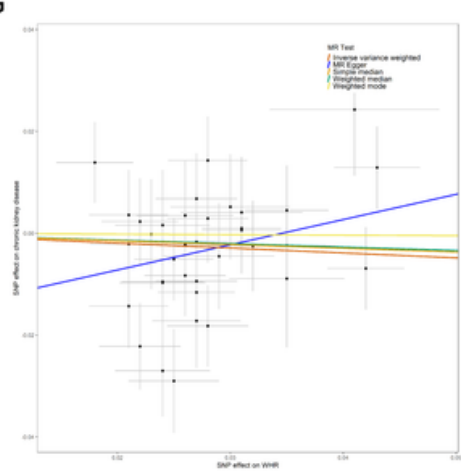

D

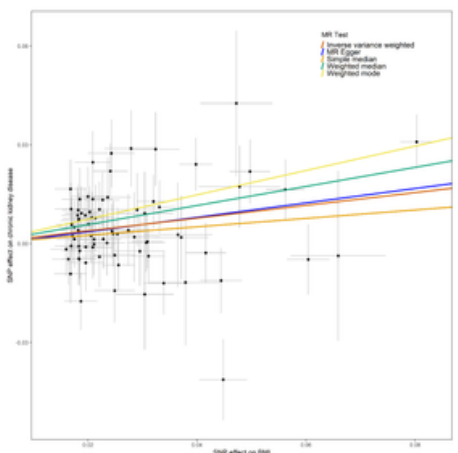

\section{Figure 2}

Scatter plot of single nucleotide polymorphism (SNP) potential effects on obesity traits versus CKD. The $95 \% \mathrm{Cl}$ for the effect size on CKD is shown as vertical lines, while the $95 \% \mathrm{Cl}$ for the effect size is shown as horizontal lines. The slope of fitted lines represents the estimated Mendelian randomization effect per method. 
A

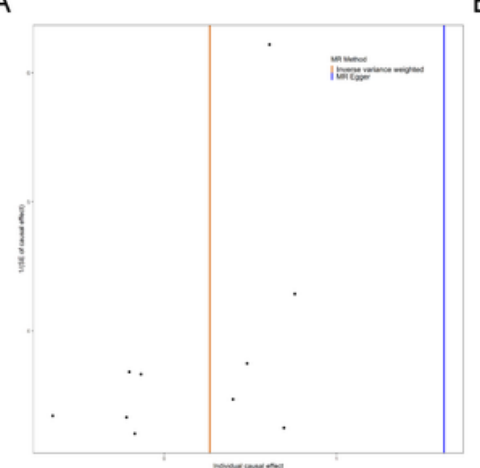

E

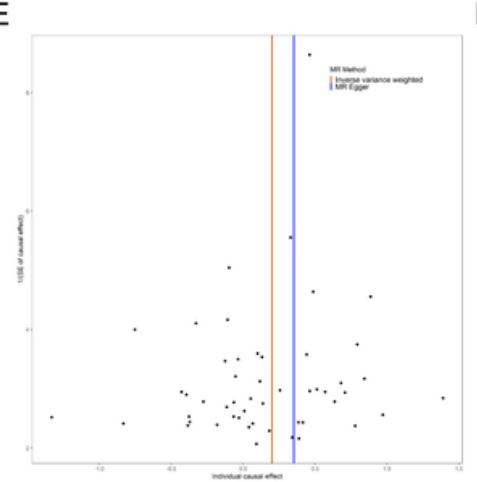

B

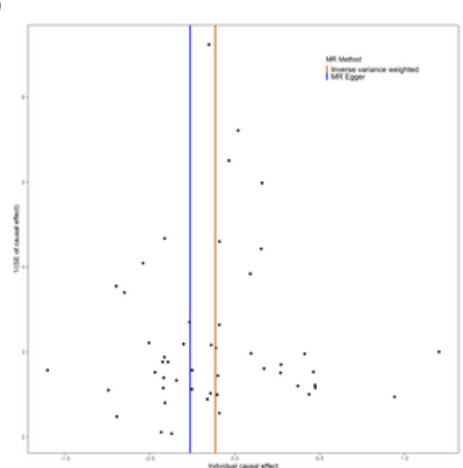

F

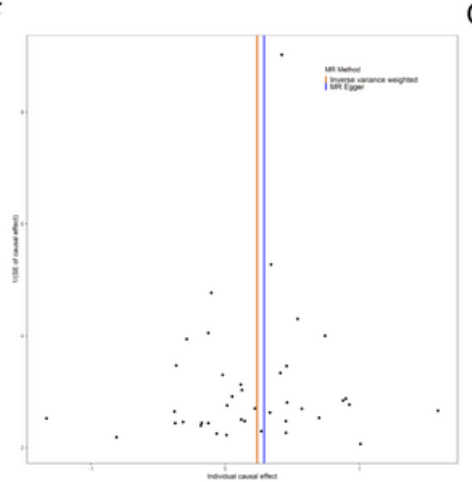

C

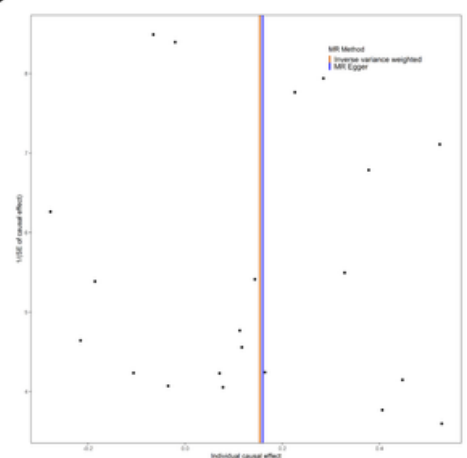

D

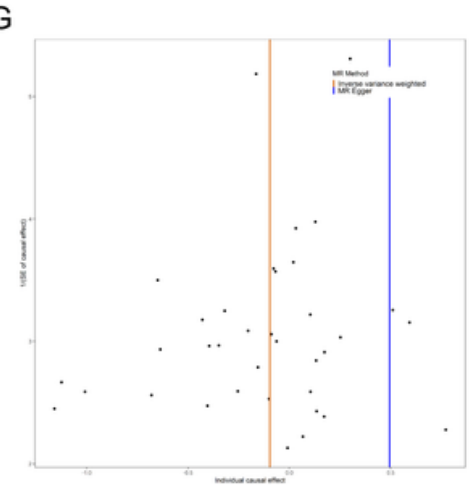

Figure 3

Funnel plot shows the estimation using the inverse of the standard error of the causal estimate with each individual SNP as a tool. The vertical line represents the estimated causal effect obtained using IVW and MR-Egger method. 